\title{
Pattern Nulling of Linear Antenna Arrays Using Backtracking Search Optimization Algorithm
}

\author{
Kerim Guney ${ }^{1}$ and Ali Durmus ${ }^{2}$ \\ ${ }^{1}$ Department of Electrical and Electronics Engineering, Faculty of Engineering, Nuh Naci Yazgan University, 38040 Kayseri, Turkey \\ ${ }^{2}$ Department of Electricity and Energy, Vocational College, Erciyes University, 38039 Kayseri, Turkey \\ Correspondence should be addressed to Ali Durmus; alidurmus@gmail.com
}

Received 14 January 2015; Accepted 27 March 2015

Academic Editor: Miguel Ferrando Bataller

Copyright (c) $2015 \mathrm{~K}$. Guney and A. Durmus. This is an open access article distributed under the Creative Commons Attribution License, which permits unrestricted use, distribution, and reproduction in any medium, provided the original work is properly cited.

\begin{abstract}
An evolutionary method based on backtracking search optimization algorithm (BSA) is proposed for linear antenna array pattern synthesis with prescribed nulls at interference directions. Pattern nulling is obtained by controlling only the amplitude, position, and phase of the antenna array elements. BSA is an innovative metaheuristic technique based on an iterative process. Various numerical examples of linear array patterns with the prescribed single, multiple, and wide nulls are given to illustrate the performance and flexibility of BSA. The results obtained by BSA are compared with the results of the following seventeen algorithms: particle swarm optimization (PSO), genetic algorithm (GA), modified touring ant colony algorithm (MTACO), quadratic programming method (QPM), bacterial foraging algorithm (BFA), bees algorithm (BA), clonal selection algorithm (CLONALG), plant growth simulation algorithm (PGSA), tabu search algorithm (TSA), memetic algorithm (MA), nondominated sorting GA-2 (NSGA-2), multiobjective differential evolution (MODE), decomposition with differential evolution (MOEA/D-DE), comprehensive learning PSO (CLPSO), harmony search algorithm (HSA), seeker optimization algorithm (SOA), and mean variance mapping optimization (MVMO). The simulation results show that the linear antenna array synthesis using BSA provides low side-lobe levels and deep null levels.
\end{abstract}

\section{Introduction}

As a result of the increase in electromagnetic pollution, array pattern nulling techniques have received a significant attention in the past decades. These techniques are used in a large number of application areas, especially in sonar, communication, and radar systems to minimize degradation in signal-to-noise ratio [1]. Pattern nulling techniques have been extensively studied in the literature [2-43]. In recent years, the metaheuristic algorithms [7-43] have received remarkable attention for the null steering in antenna array patterns. These metaheuristic algorithms are more flexible than traditional methods and do not use derivative information. Additionally, the metaheuristic algorithms are capable of escaping from local minima. Genetic algorithm (GA) [7$12,20,23,25,39]$, tabu search algorithm (TSA) [23, 30], modified touring ant colony algorithm (MTACO) [13], particle swarm optimization (PSO) $[15,30,33]$, bees algorithm (BA) $[16,29]$, bacterial foraging algorithm (BFA) [19, 24], clonal selection algorithm (CLONALG) [21], plant growth simulation algorithm (PGSA) [26], differential evolution (DE) algorithm [14, 18, 27], biogeography based optimization (BBO) [28], multiobjective DE (MODE) [30], memetic algorithm (MA) [17, 23, 30], nondominated sorting GA2 (NSGA-2) [30], decomposition with DE (MOEA/D-DE) [30], comprehensive learning PSO (CLPSO) [31], seeker optimization algorithm (SOA) [32], invasive weed optimization (IWO) algorithm [34], harmony search algorithm (HSA) [35], firefly algorithm (FA) [36, 38], cuckoo search (CS) algorithm [37, 42], differential search algorithm (DSA) [40], cat swarm optimization (CSO) [41], and mean variance mapping optimization (MVMO) [43] can be given as the examples of these metaheuristic algorithms used for pattern nulling.

In this paper, backtracking search optimization algorithm (BSA) [44] is presented for pattern nulling by controlling only the amplitude, phase, and position of the antenna 
array elements. The BSA is a metaheuristic search optimization algorithm based on an iterative process. It was introduced by Civicioglu in 2013 [44]. Contrary to many other metaheuristic algorithms, BSA has a single control parameter. Many optimization methods are very difficult and complex to understand, but BSA has a very simple structure to adapt different optimization problems. In [44], BSA was statistically compared with artificial bee colony (ABC), PSO, covariance matrix adaptation evolution strategy (CMAES), CLPSO, adaptive DE (JDE), and self-adaptive DE (SADE) algorithms. Three real world benchmark and seventy-five boundary constrained problems were used for comparison. The simulations and comparisons in [44] indicate that BSA can provide optimal solutions for benchmark problems and that the results of BSA are more efficient than the compared algorithms. BSA was used in [45] and [46] for circular and concentric circular antenna array designs, respectively. BSA was also employed for solving different optimization problems [47-51].

The study will continue in the following order. The general problem formulations of linear antenna arrays synthesis are given in Section 2. A brief explanation of the BSA is presented in Section 3. In Section 4, numerical results are given. Finally, conclusions of the paper are given in Section 5.

\section{Problem Formulation}

We assume a linear antenna array with $M$ elements located throughout the $x$-axis. The far field array factor (AF) can be indicated as

$$
\operatorname{AF}(\theta)=\sum_{m=1}^{M} I_{m} e^{j\left(k d_{m} \sin \theta+\delta_{m}\right)}
$$

where $k$ is the number of wave $(k=2 \pi / \lambda), \theta$ is the angle of scanning, and $d_{m}, \delta_{m}$, and $I_{m}$ are the location, phase, and amplitude of the $m$ th element, respectively.

When the linear antenna array elements are located symmetrically and excited around the array center and the number of antenna array elements is even $N=M / 2$, it is possible to indicate array factor in the following way:

$$
\operatorname{AF}(\theta)=2 \sum_{m=1}^{N} I_{m} \cos \left(k d_{m} \cos \theta+\delta_{m}\right) .
$$

The main aim of the study is to find a set of optimum element amplitude, phase, or position values in order to produce a radiation pattern with low maximum side-lobe level (MSL) and deep nulls placed in desired directions. For this reason, the following cost function will be minimized by BSA:

$$
C=\sum_{\theta=-90^{\circ}}^{90^{\circ}}\left[W(\theta)\left|\mathrm{AF}_{o}(\theta)-\mathrm{AF}_{d}(\theta)\right|+\operatorname{ESL}(\theta)\right],
$$

where $\operatorname{AF}_{o}(\theta)$ is the array factor value achieved by BSA and $\operatorname{AF}_{d}(\theta)$ is the desired array factor value. $W(\theta)$ and $\operatorname{ESL}(\theta)$ are utilized for controlling the null depth level (NDL) and the MSL, respectively.

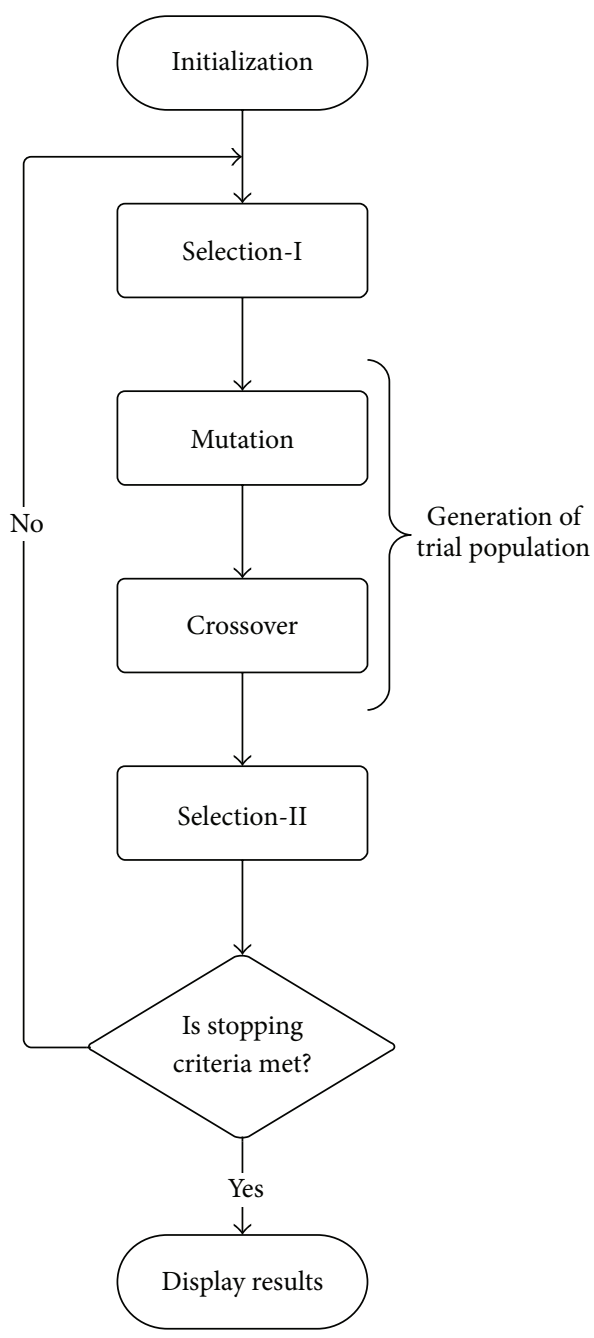

Figure 1: General flow chart of BSA.

\section{Backtracking Search Optimization Algorithm (BSA)}

BSA is an innovative metaheuristic algorithm to solve different complex and nonlinear optimization problems [44]. BSA is based on an iterative process which finds global minimum in the solution space. BSA has five evolutionary steps including initialization, selection-I, mutation, crossover, and selection-II. The structure of BSA is quite simple, so it can be applied to different complex optimization problems with ease. The general structure of BSA is shown in Figure 1.

3.1. Initialization. The initial population $(P)$ is produced by utilizing a uniform random distribution in the solution space. The following equation is used to indicate the population members:

$$
\begin{aligned}
& P_{i, j} \sim U\left(\text { low }_{j}, \mathrm{up}_{j}\right), \\
& j=1,2,3, \ldots, D, \quad i=1,2,3, \ldots, N,
\end{aligned}
$$

where $D$ is the problem dimension, $N$ is the population size, and $\operatorname{low}_{j}$ and $\mathrm{up}_{j}$ are lower and upper values of optimization problems, respectively. 
3.2. Selection-I. BSA generates the historical population $(\operatorname{old} P)$ with the calculation of the search direction in the selection-I stage. The historical population's initial values are determined by

$$
\operatorname{old} P_{i, j} \sim U\left(\operatorname{low}_{j}, \operatorname{up}_{j}\right) .
$$

Equation (6) enables BSA to redefine the historical population at the beginning of each iteration:

$$
\text { if } a<b \text { then old } P:=P \mid a, b \sim U(0,1) \text {, }
$$

where $a$ and $b$ are the uniform real numbers in the range of $[0,1]$. The following equation is used to change the arrangement of the members randomly in historical population by using shuffling function:

$$
\operatorname{old} P:=\text { permuting }(\operatorname{old} P),
$$

where the permuting() function represents a random shuffling function.

3.3. Mutation. In the mutation process, mutants, initial form of the trial populations, are generated by

$$
\text { Mutant }=P+F \cdot(\text { old } P-P),
$$

where $F$ is scale value to control the amplitude of the direction of search. BSA produces a trial population depending on old $P$ values.

3.4. Crossover. In the crossover step of BSA, final form of the trial-population $T$ is produced. The crossover process involves two steps. The first step uses mix-rate and the second step imposes that just one arbitrary individual mutates in each trial.

3.5. Selection-II. All trial populations $T_{i} s$ with optimal values better than the relevant $P_{i} s$ are utilized to update the $P_{i} s$ by using the greedy selection strategy. If $P_{\text {best }}$ 's value is better than the global minimum value, the new global minimum value is changed with the best individual of $P$. Further details of the BSA can be found in [44].

\section{Numerical Results}

To show the efficiency and performance of the BSA for steering single, multiple, and wide nulls with the prescribed directions by controlling amplitude only, phase only, and position only, eleven examples of a linear antenna array have been performed. In the first eight examples, a $30 \mathrm{~dB}$ Chebyshev pattern, having 20 equispaced elements with $0.5 \lambda$ interelement spacing, is utilized as the initial radiation pattern, as shown in Figure 2. In the last three examples, the initial pattern is chosen as a uniform array pattern for 22,28 , and 32 equispaced elements with $0.5 \lambda$ interelement spacing. The main aim is to obtain antenna array patterns having deep nulls at desired directions and low MSLs. The dynamic range ratio $\left(\mathrm{DRR}=\left|I_{\max } / I_{\min }\right|\right)$ is also taken into account for practical considerations.

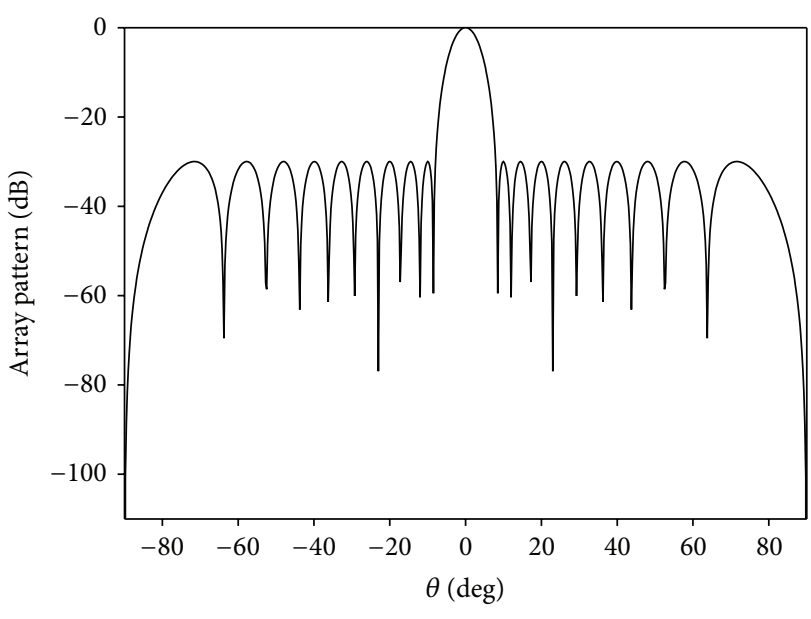

FIGURE 2: Initial Chebyshev pattern.

The maximum iteration number of the BSA is selected as 10.000. The mix-rate and step size amplification parameter of BSA are set to 1 and $F=3 \cdot$ rndn, where rndn $\sim N(0,1)$, respectively. The population size is fixed to 30 . The simulations are carried on a computer which has $2.8 \mathrm{GHz}$ i7 processor and $4 \mathrm{~GB}$ RAM. The software used for the optimization applications of BSA is MATLAB.

In this study, comprehensive comparisons have been presented. The synthesis results of BSA are compared with the results of seventeen different algorithms, PSO $[15,30]$, GA [30], MTACO [13], QPM [15], BFA [19, 24], BA [16, 29], CLONALG [21], PGSA [26], TSA [30], MA [30], NSGA-2 [30], MODE [30], MOEA/D-DE [30], CLPSO [31], SOA [32], HSA [35], and MVMO [43].

In the first group of examples, BSA is used to achieve radiation patterns with imposed nulls at interference directions by controlling the amplitude only. In the second group of examples, desired nulls are placed on the array pattern by controlling phase only. In the third group of examples, only the element positions are controlled for pattern nulling.

4.1. Amplitude Only Control. In the first example, Chebyshev pattern is considered with a single null at $\theta_{i}=14^{\circ}$. The parameters values of the cost function are selected as

$$
\begin{gathered}
A_{d}(\theta)= \begin{cases}0, & \text { for } \theta=\theta_{i} \\
\text { Initial pattern, } & \text { elsewhere, }\end{cases} \\
W(\theta)= \begin{cases}150, & \text { for } \theta=\theta_{i} \\
1, & \text { elsewhere, }\end{cases} \\
\operatorname{ESL}(\theta)= \begin{cases}80, & \text { if MSL }>-29.1 \mathrm{~dB} \\
0, & \text { elsewhere. }\end{cases}
\end{gathered}
$$

The radiation pattern achieved by BSA is shown in Figure 3. Table 1 illustrates NDL, MSL, and DRR values of the radiation pattern obtained by using BSA. Table 1 also shows the results of MTACO [13], BA [16], PGSA [26], HSA [35], and BFA [19]. It can be clearly observed from Table 1 that NDL, MSL, and 
TABLE 1: NDL, MSL, and DRR values obtained by BSA and other optimization techniques MTACO [13], BA [16], PGSA [26], HSA [35], and BFA [19] for one null imposed at $14^{\circ}$.

\begin{tabular}{lcccccc}
\hline & BSA (Figure 3) & MTACO [13] & BA [16] & PGSA [26] & HSA [35] & BFA [19] \\
\hline NDL (dB) & -134.2 & -88.1 & -100.4 & -118.3 & -122.5 & -113.6 \\
MSL (dB) & -29.22 & -29.10 & -28.80 & -28.03 & -29.14 & 3.87 \\
DRR & 3.86 & 4.36 & 4.09 & 3.89 & 3.89 \\
\hline
\end{tabular}

TABLE 2: The element amplitudes $\left(I_{m}\right)$ for the array patterns given in Figures 2,3 , and 5-8 and the phases $\left(\delta_{k}\right)$ in degree for the array patterns given in Figures 9-11.

\begin{tabular}{|c|c|c|c|c|c|c|c|c|c|}
\hline \multirow{2}{*}{$m$} & \multirow{2}{*}{$\begin{array}{c}\text { Initial } \\
\text { Chebyshev } \\
\text { pattern } \\
\text { Figure } 2\left(I_{m}\right)\end{array}$} & \multicolumn{8}{|c|}{ Computed with BSA } \\
\hline & & Figure $3\left(I_{m}\right)$ & Figure $5\left(I_{m}\right)$ & Figure $6\left(I_{m}\right)$ & Figure $7\left(I_{m}\right)$ & Figure $8\left(I_{m}\right)$ & Figure $9\left(\delta_{k}\right)$ & Figure $10\left(\delta_{k}\right)$ & Figure $11\left(\delta_{k}\right)$ \\
\hline \pm 1 & 1.00000 & 1.00000 & 1.00000 & 0.84376 & 0.98752 & 1.00000 & 0.94847 & -1.15397 & -0.37393 \\
\hline \pm 2 & 0.97010 & 0.98714 & 0.93080 & 0.85439 & 1.00000 & 0.94790 & 3.35183 & -3.36187 & -3.96817 \\
\hline \pm 3 & 0.91243 & 0.94987 & 0.89094 & 0.85078 & 0.99002 & 0.93151 & 5.70358 & -5.59187 & -8.10346 \\
\hline \pm 4 & 0.83102 & 0.88163 & 0.83167 & 0.74231 & 0.85050 & 0.88528 & 7.59405 & -5.05200 & -5.63161 \\
\hline \pm 5 & 0.73147 & 0.77212 & 0.74139 & 0.60989 & 0.74589 & 0.63226 & 9.32972 & -7.11779 & -3.07266 \\
\hline \pm 6 & 0.62034 & 0.63944 & 0.60244 & 0.55080 & 0.60333 & 0.60860 & 10.55083 & -12.00020 & -12.00112 \\
\hline \pm 7 & 0.50461 & 0.48298 & 0.45724 & 0.44059 & 0.36134 & 0.49878 & 10.51685 & -18.81668 & -21.61602 \\
\hline \pm 8 & 0.39104 & 0.33060 & 0.31903 & 0.30532 & 0.46506 & 0.41636 & 9.93409 & -24.08489 & -18.97410 \\
\hline \pm 9 & 0.28558 & 0.25882 & 0.22003 & 0.17434 & 0.20647 & 0.24505 & 8.38402 & -33.72318 & -29.99998 \\
\hline \pm 10 & 0.32561 & 0.29505 & 0.23683 & 0.22392 & 0.15120 & 0.19214 & 3.60727 & -14.86452 & -30.00000 \\
\hline
\end{tabular}

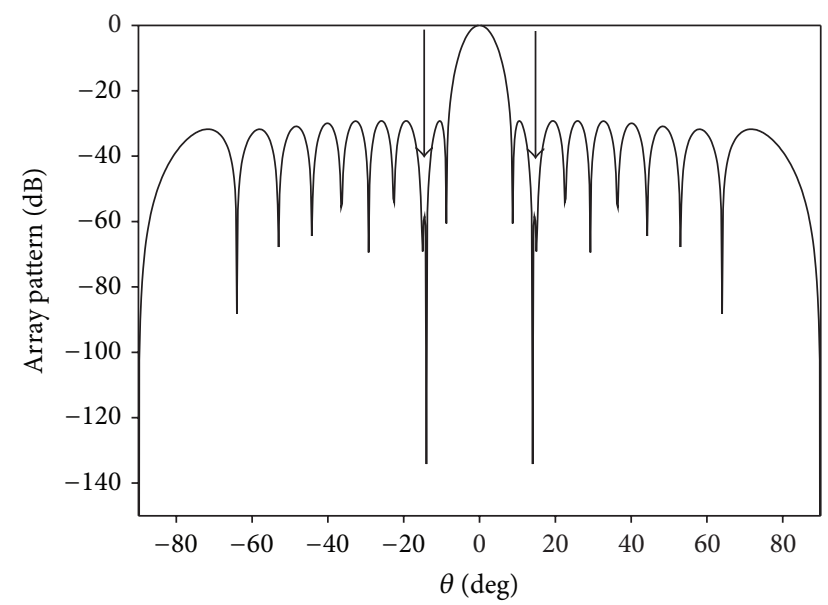

FIGURE 3: Radiation pattern achieved by controlling the amplitude only with one null at $14^{\circ}$.

DRR values of BSA are better than those of MTACO [13], BA [16], PGSA [26], HSA [35], and BFA [19].

Figure 4 shows the convergence curve. The curve is the average values obtained by running the algorithm 30 times. It is apparent from this figure that 1000 iterations are needed to find the optimal solutions.

To illustrate the resilience of BSA, in the second example, $W(\theta)$ is redefined as follows:

$$
W(\theta)= \begin{cases}250, & \text { for } \theta=\theta_{i} \\ 1, & \text { elsewhere }\end{cases}
$$

Figure 5 shows the radiation pattern produced by the BSA. The NDL and MSL values of the radiation pattern in Figure 5 are $-182.3 \mathrm{~dB}$ and $-29.08 \mathrm{~dB}$, respectively. In Figure 5, the NDL value of array pattern is better than the NDL value of the pattern in Figure 3. However, compared to the improvement in the NDL, the MSL value of the radiation pattern in Figure 5 is seen worse than the MSL of the pattern in Figure 3. There is a trade-off between the NDL and MSL; usually, performance cannot be improved considerably for one without sacrificing the other.

In third and fourth examples, to test the capability of BSA for the radiation pattern synthesis with multiple nulls at any direction, only the $\operatorname{AF}_{d}(\theta)$ given by (9) is changed for synthesizing the array patterns with double null at $14^{\circ}$ and $26^{\circ}$ and triple null at $14^{\circ}, 26^{\circ}$, and $33^{\circ}$, respectively. Figures 6 and 7 show the radiation patterns obtained by BSA for the multiple nulls, as it is seen from Figures 6 and 7 that each imposed null achieved by BSA is lower than $-120 \mathrm{~dB}$.

In the fifth example, the radiation pattern with a wide null placed at $30^{\circ}$ with $\Delta \theta=5^{\circ}$ is obtained and is illustrated in Figure 8 . The NDL value of the radiation pattern achieved by BSA is deeper than $-59 \mathrm{~dB}$.

The normalized amplitude values of array elements for the radiation patterns showed in Figures 2, 3, and 5-8 are given in Table 2. It is clearly seen that, with respect to the main beam, the radiation patterns in Figures 3 and 5-8 are symmetric. This is a result of the even symmetry of the element amplitudes around the array center. For this reason, while a null occurs at the one side of the main beam, an image null imposes at the other side. 


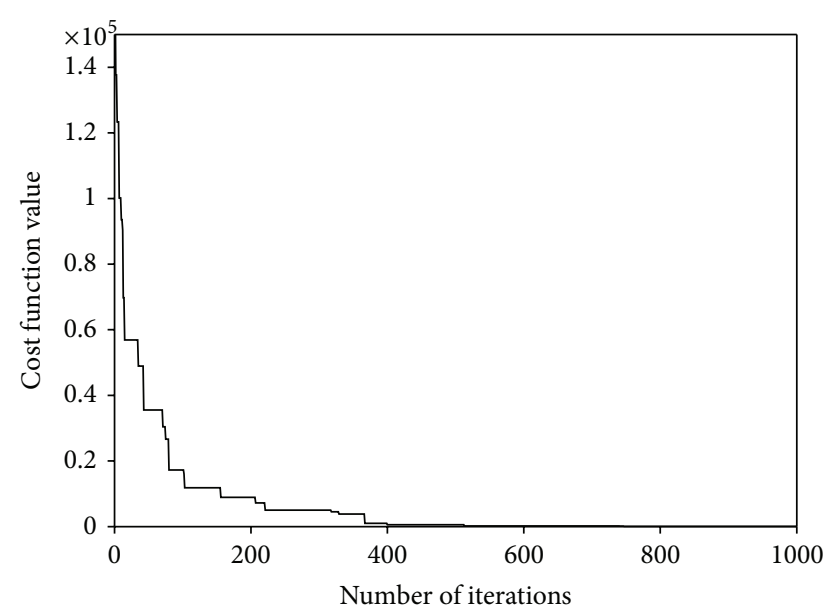

FIGURE 4: Convergence curve of the nulling pattern achieved by the BSA.

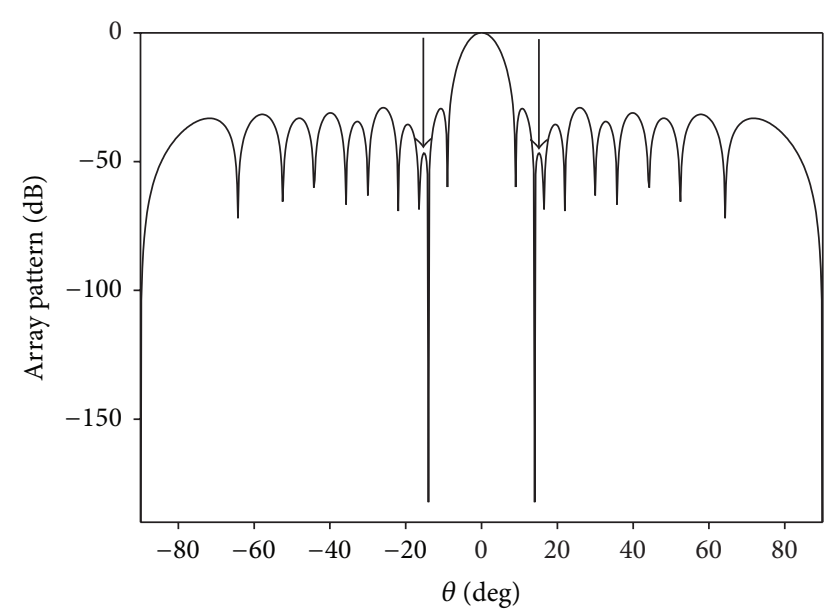

FIGURE 5: Radiation pattern achieved by controlling the amplitude only with a null depth level deeper than that of the first example having one null at $14^{\circ}$.

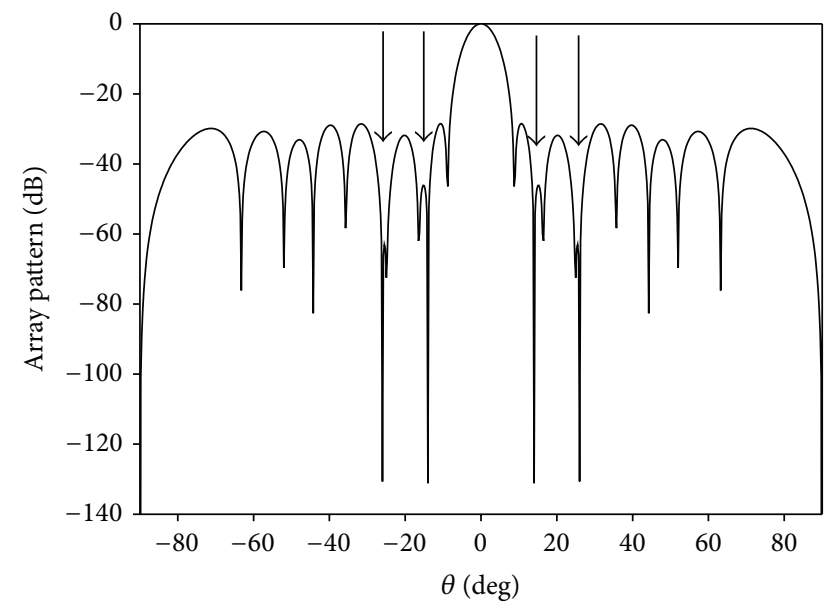

FIGURE 6: Radiation pattern obtained by controlling the amplitude only with double null at $14^{\circ}$ and $26^{\circ}$.

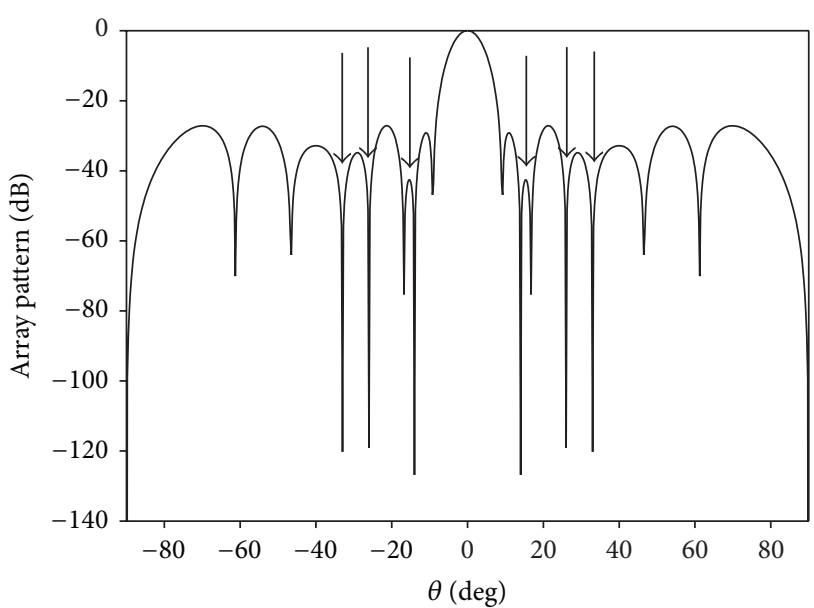

FIGURE 7: Radiation pattern obtained by controlling the amplitude only with triple null at $14^{\circ}, 26^{\circ}$, and $33^{\circ}$.

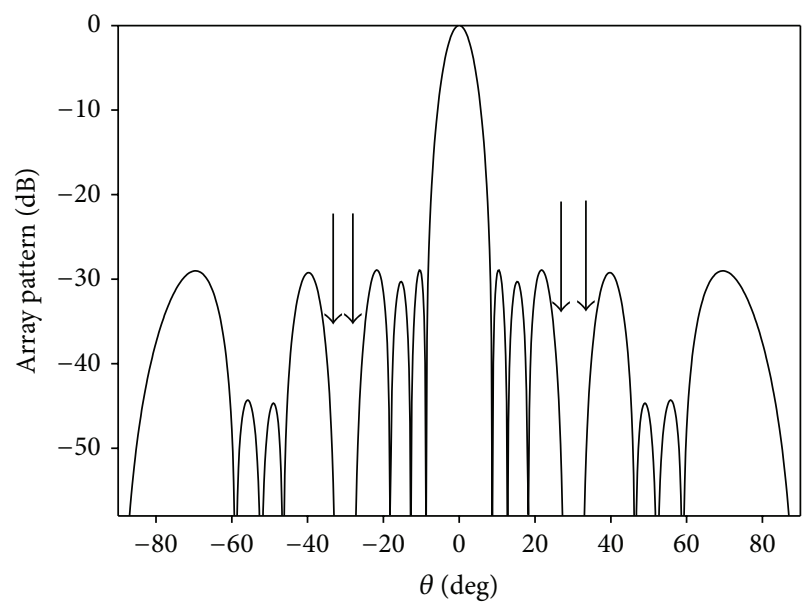

FIGURE 8: Radiation pattern achieved by controlling the amplitude only with a wide null sector centered $30^{\circ}$ with $\Delta \theta=5^{\circ}$.

4.2. Phase Only Control. In the next three examples, forming nulls in the radiation pattern is achieved by controlling only the phase of antenna array element. The elements amplitude values $\left(I_{m}\right)$ of these examples are the same as the values of initial Chebyshev array.

In the sixth example, the null direction $\theta_{i}$ is identified as $-10^{\circ}$. The radiation pattern obtained by BSA is given in Figure 9. In Table 3, the NDL and MSL values of the radiation pattern obtained by BSA are compared with those of the CLONALG [21], BA [29], HSA [35], and BFA [24]. As it can be seen from this table, the NDL and MSL values of the pattern obtained by BSA are better than the other compared algorithms.

Double null at $\theta_{i 1}=-10^{\circ}$ and $\theta_{i 2}=20^{\circ}$ is achieved by controlling only the phase of the antenna array elements in the seventh example. The radiation pattern obtained by BSA is given in Figure 10. The MSL and NDL values of the radiation pattern obtained by BSA are compared with the results of SOA [32], CLONALG [21], and BFA [24] in Table 4. 
TABLE 3: NDL and MSL values obtained by BSA and other optimization techniques CLONALG [21], BA [29], HSA [35], and BFA [24] for one null imposed at $-10^{\circ}$.

\begin{tabular}{lccccc}
\hline & BSA (Figure 9) & CLONALG [21] & BA [29] & HSA [35] & BFA [24] \\
\hline NDL (dB) & -212.30 & -175 & -185 & -202 & -161 \\
MSL (dB) & -24.47 & -24.0 & -24.22 & -24.23 & -24.0 \\
\hline
\end{tabular}

TABLE 4: MSL and NDL values obtained by BSA and other optimization techniques SOA [32], CLONALG [21], and BFA [24] for double null imposed at $-10^{\circ}$ and $20^{\circ}$.

\begin{tabular}{|c|c|c|c|c|}
\hline & BSA (Figure 10) & SOA [32] & CLONALG [21] & BFA [24] \\
\hline MSL (dB) & -24.47 & -23.96 & -24.05 & -24.28 \\
\hline $\mathrm{NDL}$ at $-10^{\circ}(\mathrm{dB})$ & -177.50 & -119.80 & -154.20 & -156.30 \\
\hline $\mathrm{NDL}$ at $20^{\circ}(\mathrm{dB})$ & -188.50 & -112.60 & -156.50 & -187.60 \\
\hline
\end{tabular}

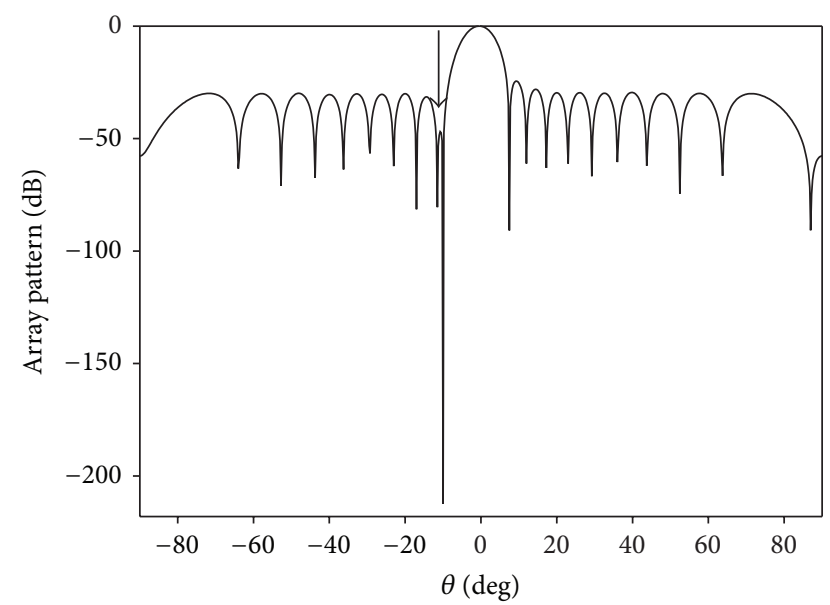

FIGURE 9: Radiation pattern found by phase only control with one null at $-10^{\circ}$.

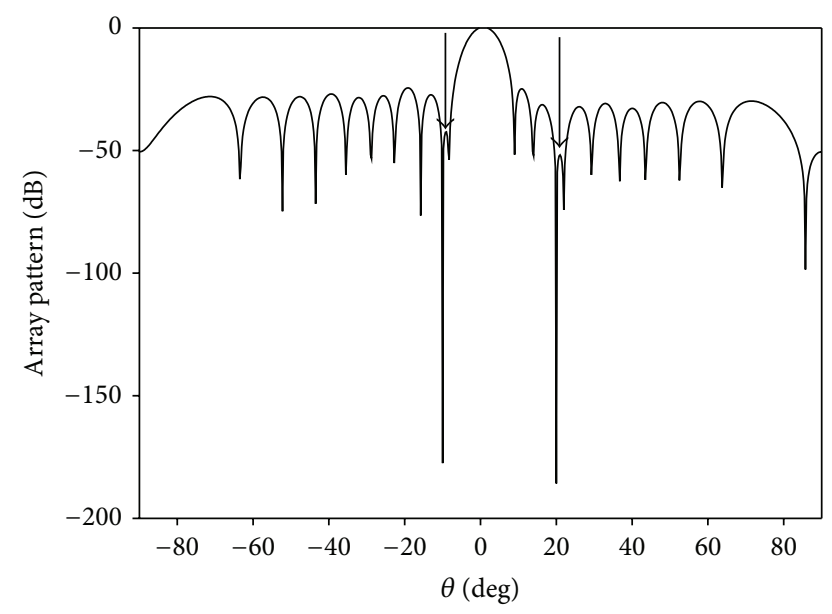

FIGURE 10: Radiation pattern found by phase only control with double null at $-10^{\circ}$ and $20^{\circ}$.

It is possible to see from Table 4 that the results of BSA are better than those of other optimization methods.

In the eighth example, the radiation pattern with triple null $\left(\theta_{i 1}=-10^{\circ}, \theta_{i 2}=20^{\circ}\right.$, and $\left.\theta_{i 3}=-33^{\circ}\right)$ is achieved by BSA and is illustrated in Figure 11. In Table 5, the MSL

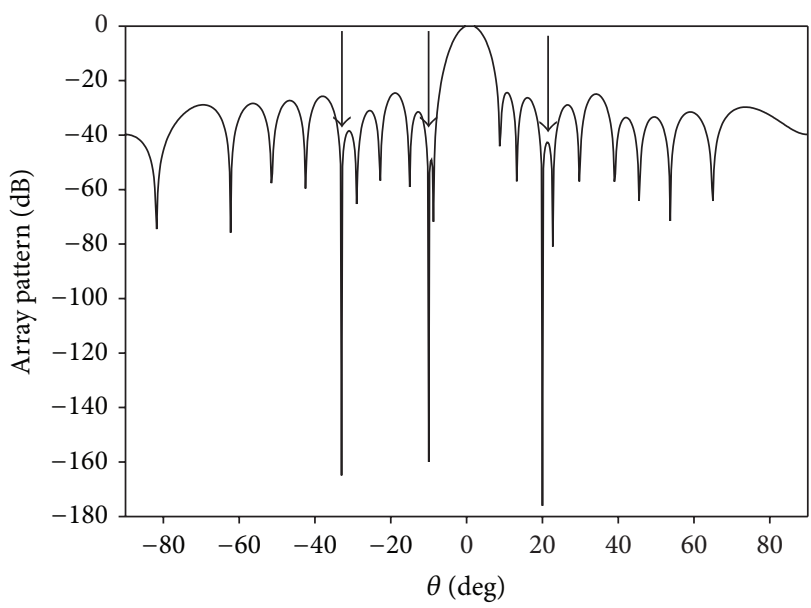

FIGURE 11: Radiation pattern found by phase only control with triple null at $-33^{\circ},-10^{\circ}$, and $20^{\circ}$.

and NDL values of the radiation pattern achieved by BSA are compared with the MSL and NDL values of the radiation patterns achieved by BA [29], CLONALG [21], and SOA [32]. It is clearly seen from Table 5 that MSL and NDL values of the pattern obtained by BSA are better than those of BA [29], CLONALG [21], and SOA [32] except that NDL value of CLONALG at $-10^{\circ}$ is deeper than NDL value of BSA.

The phase values of the array elements for the patterns obtained by BSA in Figures 9-11 are given in Table 2.

4.3. Position Only Control. In the remaining three examples, nulls in the radiation pattern are obtained by controlling only the positions of uniform linear antenna array elements. The ninth example shows the synthesis of a 22-element antenna array for low MSL with desired null at $9^{\circ}$. In Figure 12, the radiation pattern obtained by BSA is illustrated. The NDL and MSL values of the radiation pattern obtained from BSA are given in Table 6 . Table 6 also shows the NDL and MSL results of TSA [30], GA [30], MA [30], HSA [35], PSO [30], NSGA-2 [30], MODE [30], and MOEA/D-DE [30] for a comparison. According to Table 6, it is evidently seen that the NDL and MSL values of the radiation pattern obtained from BSA are better than those of other metaheuristic algorithms. 
TABLE 5: MSL and NDL values obtained by BSA and other optimization techniques BA [29], CLONALG [21], and SOA [32] for triple null imposed at $-33^{\circ},-10^{\circ}$, and $20^{\circ}$.

\begin{tabular}{|c|c|c|c|c|}
\hline & BSA (Figure 11) & BA [29] & CLONALG [21] & SOA [32] \\
\hline MSL (dB) & -24.40 & -24.18 & -23.46 & -23.72 \\
\hline $\mathrm{NDL}$ at $-33^{\circ}(\mathrm{dB})$ & -164.90 & -157.60 & -151.00 & -117.10 \\
\hline $\mathrm{NDL}$ at $-10^{\circ}(\mathrm{dB})$ & -160.00 & -156.10 & -178.80 & -111.10 \\
\hline $\mathrm{NDL}$ at $20^{\circ}(\mathrm{dB})$ & -176.10 & -163.90 & -135.30 & -133.40 \\
\hline
\end{tabular}

TABLE 6: NDL and MSL values obtained by BSA and other optimization techniques TSA [30], GA [30], MA [30], HSA [35], PSO [30], NSGA-2 [30], MODE [30], and MOEA/D-DE [30] for 22-element linear antenna array with imposed one null at $9^{\circ}$.

\begin{tabular}{lccccccccc}
\hline & BSA (Figure 12) & TSA [30] & GA [30] & MA [30] & HSA [35] & PSO [30] & NSGA-2 [30] & MODE [30] & MOEA/D-DE [30] \\
\hline NDL (dB) & -104.61 & -67.94 & -54.29 & -73.92 & -103.30 & -49.94 & -58.79 & -78.78 & -69.64 \\
MSL (dB) & -23.54 & -17.17 & -15.73 & -18.11 & -23.28 & -20.68 & -17.27 & -18.05 & -20.93 \\
\hline
\end{tabular}

TABLE 7: MSL and NDL values obtained by BSA and other optimization techniques CLPSO [31] and MVMO [43] for 28-element linear antenna array with imposed triple null at $30^{\circ}, 32.5^{\circ}$, and $35^{\circ}$.

\begin{tabular}{lccc}
\hline & BSA (Figure 13) & CLPSO [31] & MVMO [43] \\
\hline MSL $(\mathrm{dB})$ & -21.90 & -21.63 & -21.63 \\
NDL at $30^{\circ}(\mathrm{dB})$ & -82.49 & -60.04 & -70.19 \\
NDL at $32.5^{\circ}(\mathrm{dB})$ & -93.59 & -60.01 & -78.79 \\
NDL at $35^{\circ}(\mathrm{dB})$ & -80.49 & -60.00 & -71.79 \\
\hline
\end{tabular}

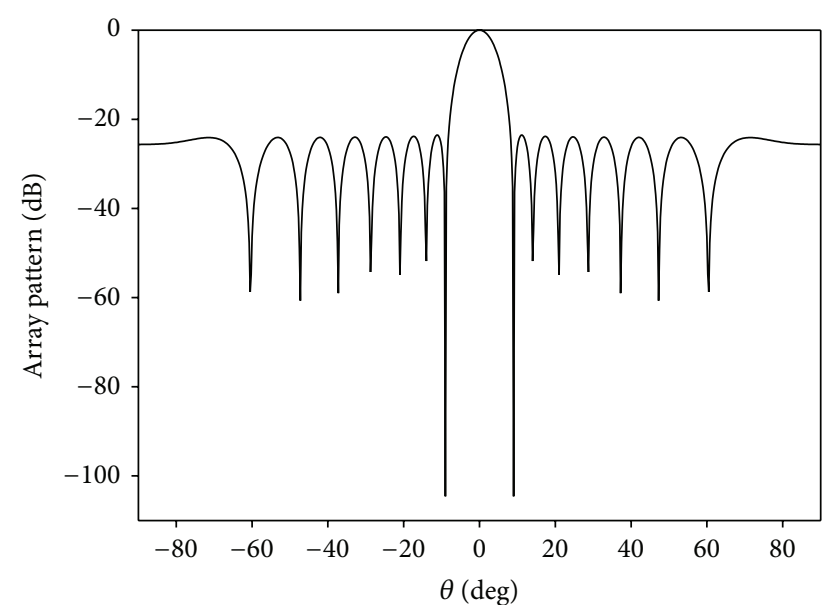

FIGURE 12: 22-element array pattern found by position only control with one imposed null at $9^{\circ}$.

In the tenth example, BSA is utilized to synthesize a 28element linear antenna array which has a pattern with low MSL and nulls at $30^{\circ}, 32.5^{\circ}$, and $35^{\circ}$. The radiation pattern generated by BSA is illustrated in Figure 13. In Table 7, the MSL and NDL values of the radiation pattern of BSA are compared with the results of CLPSO [31] and MVMO [43]. It can be seen from Table 7 that the results of BSA are better than those of CLPSO [31] and MVMO [43]. It was emphasized in [31] that the performance of CLPSO is better than the common PSO algorithms and a real-coded GA.

In the last example, BSA is performed to synthesize 32element linear antenna array that has a null at the direction

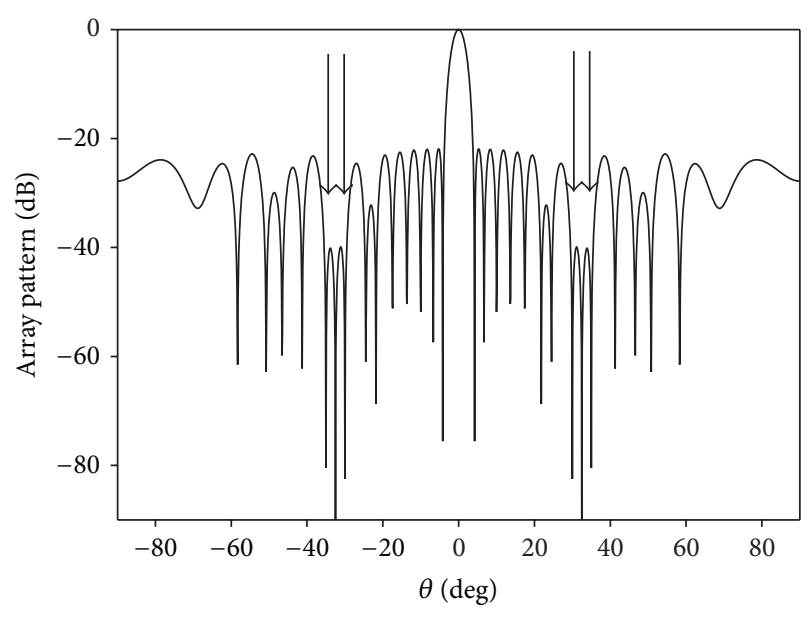

FIGURE 13: 28-element array pattern found by position only control with triple null at $30^{\circ}, 32.5^{\circ}$, and $35^{\circ}$.

of $9^{\circ}$ and low MSL. The radiation pattern achieved by BSA is given in Figure 14. In Table 8, NDL and MSL values of the pattern obtained by BSA are compared with NDL and MSL values of the patterns obtained by PSO [15], HSA [35], and QPM [15]. It is apparent from Table 8 that NDL and MSL values of the radiation pattern obtained by BSA are better than those of PSO [15], HSA [35], and QPM [15].

The position values of the antenna array elements calculated by BSA for the radiation patterns in Figures 12-14 are given in Table 9. 
TABLE 8: NDL and MSL values obtained by BSA and other optimization techniques PSO [15], HSA [35], and QPM [15] for 32-element linear antenna array with imposed one null at $9^{\circ}$.

\begin{tabular}{lcccc}
\hline & BSA (Figure 14) & PSO [15] & HSA [35] & QPM [15] \\
\hline NDL (dB) & -107.50 & -62.06 & -88.08 & -34.74 \\
MSL (dB) & -20.50 & -18.71 & -19.51 & -17.73 \\
\hline
\end{tabular}

TABLE 9: The element positions $\left(d_{m}\right)$ normalized with respect to $0.5 \lambda$ for the array patterns given in Figures $12-14$.

\begin{tabular}{ll}
\hline$M$ (element number) & {$\left[d_{1}, d_{2}, d_{3}, \ldots, d_{N}\right]$} \\
\hline 22 (Figure 12) & {$[0.41549,0.83227,1.21361,2.03820,2.52836,3.06061,3.88902,4.53114,5.41274,6.51612,7.62000]$} \\
\hline \multirow{2}{*}{28 (Figure 13) } & {$[0.42184,1.46852,2.22411,3.20133,4.01764,5.15375,6.24390,7.12942,8.34925,9.59401,10.98474$,} \\
& $12.75861,14.28949,15.86988]$ \\
\hline \multirow{2}{*}{32 (Figure 14) } & {$[0.56084,1.38547,2.10149,2.99246,3.61791,4.46921,5.37792,6.18462,6.98827,7.68024,8.61828$,} \\
& $9.56573,11.02684,12.53179,14.41787,15.55000]$ \\
\hline
\end{tabular}

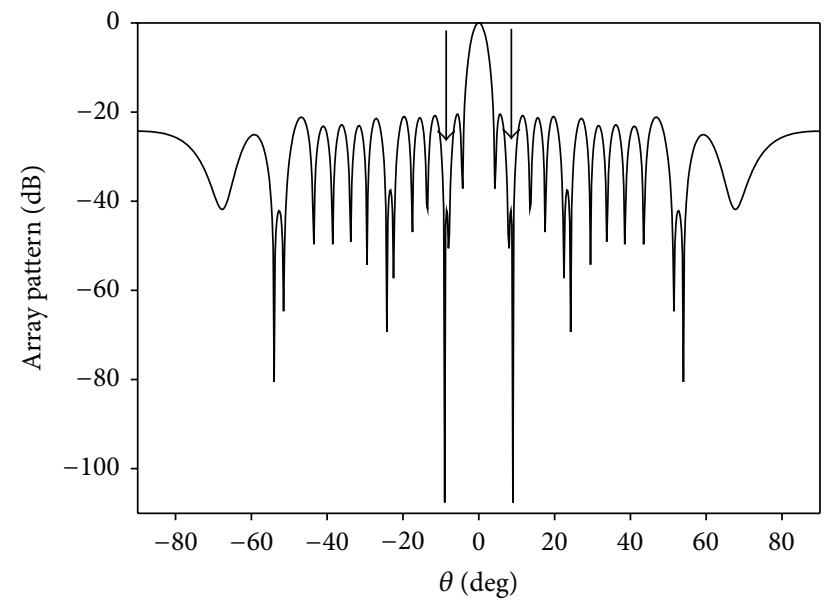

FIGURE 14: 32-element array pattern found by position only control with one imposed null at $9^{\circ}$.

\section{Conclusions}

BSA is successfully used for synthesizing linear antenna arrays with single, multiple, and wide nulls imposed at the directions of interferences. The pattern nulling is achieved by controlling the amplitude only, the phase only, and the position only. The comparisons made between the results obtained by BSA and seventeen different algorithms show that BSA is very competitive algorithm for the pattern nulling. BSA exhibits good performance in terms of MSL and NDL as compared to other seventeen algorithms. Thus, BSA has a good potential for solving other electromagnetic problems.

\section{Conflict of Interests}

The authors declare that there is no conflict of interests regarding the publication of this paper.

\section{References}

[1] R. J. Mailloux, Phased Array Antenna Handbook, Artech House, Boston, Mass, USA, 2005.
[2] R. A. Shore, "Nulling at symmetric pattern location with phase-only weight control," IEEE Transactions on Antennas and Propagation, vol. AP-32, no. 5, pp. 530-533, 1984.

[3] H. Steyskal, R. A. Shore, and R. L. Haupt, "Methods for null control and their effects on the radiation pattern," IEEE Transactions on Antennas and Propagation, vol. 34, no. 3, pp. 404-409, 1986.

[4] M. H. Er, "Linear antenna array pattern synthesis with prescribed broad nulls," IEEE Transactions on Antennas and Propagation, vol. 38, no. 9, pp. 1496-1498, 1990.

[5] H. M. Ibrahim, "Null steering by real-weight control-a method of decoupling the weights," IEEE Transactions on Antennas and Propagation, vol. 39, no. 11, pp. 1648-1650, 1991.

[6] T. H. Ismail and M. M. Dawoud, "Null steering in phased arrays by controlling the element positions," IEEE Transactions on Antennas and Propagation, vol. 39, no. 11, pp. 1561-1566, 1991.

[7] A. Tennant, M. M. Dawoud, and A. P. Anderson, "Array pattern nulling by element position perturbations using a genetic algorithm," Electronics Letters, vol. 30, no. 3, pp. 174-176, 1994.

[8] R. L. Haupt, "Phase-only adaptive nulling with a genetic algorithm," IEEE Transactions on Antennas and Propagation, vol. 45, no. 6, pp. 1009-1015, 1997.

[9] W.-P. Liao and F.-L. Chu, "Array pattern nulling by phase and position perturbations with the use of the genetic algorithm," Microwave and Optical Technology Letters, vol. 15, no. 4, pp. 251256, 1997.

[10] F. J. Ares-Pena, J. A. Rodriguez-Gonzalez, E. Villanueva-Lopez, and S. R. Rengarajan, "Genetic algorithms in the design and optimization of antenna array patterns," IEEE Transactions on Antennas and Propagation, vol. 47, no. 3, pp. 506-510, 1999.

[11] W. P. Liao and F. L. Chu, "Array pattern synthesis with null steering using genetic algorithms by controlling only the current amplitudes," International Journal of Electronics, vol. 86, no. 4, pp. 445-457, 1999.

[12] Y. C. Chung and R. L. Haupt, "Amplitude and phase adaptive nulling with a genetic algorithm," Journal of Electromagnetic Waves and Applications, vol. 14, no. 5, pp. 631-649, 2000.

[13] N. Karaboga, K. Güney, and A. Akdagli, "Null steering of linear antenna arrays with use of modified touring ant colony optimization algorithm," International Journal of RF and Microwave Computer-Aided Engineering, vol. 12, no. 4, pp. 375-383, 2002.

[14] S. Yang, Y. B. Gan, and A. Qing, "Antenna-array pattern nulling using a differential evolution algorithm," International Journal 
of RF and Microwave Computer-Aided Engineering, vol. 14, no. 1, pp. 57-63, 2004.

[15] M. M. Khodier and C. G. Christodoulou, "Linear array geometry synthesis with minimum sidelobe level and null control using particle swarm optimization," IEEE Transactions on Antennas and Propagation, vol. 53, no. 8, pp. 2674-2679, 2005.

[16] K. Guney and M. Onay, "Amplitude-only pattern nulling of linear antenna arrays with the use of bees algorithm," Progress in Electromagnetics Research, vol. 70, pp. 21-36, 2007.

[17] C.-H. Hsu and W.-J. Shyr, "Adaptive pattern nulling design of linear array antenna by phase-only perturbations using memetic algorithms," Communications in Numerical Methods in Engineering, vol. 24, no. 11, pp. 1121-1133, 2008.

[18] Y. Chen, S. Yang, G. Li, and Z. Nie, "Adaptive nulling in time-modulated antenna arrays," in Proceedings of the 8th International Symposium on Antennas, Propagation and EM Theory (ISAPE '08), pp. 713-716, Kunming, China, November 2008.

[19] K. Guney and S. Basbug, "Interference suppression of linear antenna arrays by amplitude-only control using a bacterial foraging algorithm," Progress in Electromagnetics Research, vol. 79, pp. 475-497, 2008.

[20] P. Sattari and N. Hejazi, "Array pattern null steering using Genetic Algorithm by element position perturbations," in Proceedings of the IEEE Canadian Conference on Electrical and Computer Engineering (CCECE '08), pp. 423-427, Niagara Falls, Canada, May 2008.

[21] K. Guney, B. Babayigit, and A. Akdagli, "Interference suppression of linear antenna arrays by phase-only control using a clonal selection algorithm," Journal of the Franklin Institute, vol. 345, no. 3, pp. 254-266, 2008.

[22] M. Wang and Z. Shen, "Nulling of antenna arrays including the mutual coupling effect," in Proceedings of the IEEE Vehicular Technology Conference (VTC '08), pp. 247-251, Marina Bay, Singapore, May 2008.

[23] Y. Cengiz and H. Tokat, "Linear antenna array design with use of genetic, memetic and tabu search optimization algorithms," Progress In Electromagnetics Research C, vol. 1, pp. 63-72, 2008.

[24] K. Guney and S. Basbug, "Phase-only pattern nulling of linear antenna arrays with the use of a bacterial foraging algorithm," Neural Network World, vol. 18, no. 4, pp. 257-273, 2008.

[25] Z. Hamici and T. H. Ismail, "Digital amplitude control for interference suppression using immunity genetic algorithm," Progress in Electromagnetics Research C, vol. 11, pp. 21-38, 2009.

[26] K. Guney, A. Durmus, and S. Basbug, "A plant growth simulation algorithm for pattern nulling of linear antenna arrays by amplitude control," Progress in Electromagnetics Research B, no. 17, pp. 69-84, 2009.

[27] N. Dib, S. K. Goudos, and H. Muhsen, "Application of Taguchi's optimization method and self-adaptive differential evolution to the synthesis of linear antenna arrays," Progress in Electromagnetics Research, vol. 102, pp. 159-180, 2010.

[28] U. Singh, H. Kumar, and T. S. Kamal, "Linear array synthesis using biogeography based optimization," Progress In Electromagnetics Research M, vol. 11, pp. 25-36, 2010.

[29] K. Guney and M. Onay, "Bees algorithm for interference suppression of linear antenna arrays by controlling the phaseonly and both the amplitude and phase," Expert Systems with Applications, vol. 37, no. 4, pp. 3129-3135, 2010.
[30] S. Pal, B.-Y. Qu, S. Das, and P. N. Suganthan, "Optimal synthesis of linear antenna arrays with multi-objective differential evolution," Progress in Electromagnetics Research B, vol. 21, pp. 87-111, 2010.

[31] S. K. Goudos, V. Moysiadou, T. Samaras, K. Siakavara, and J. N. Sahalos, "Application of a comprehensive learning particle swarm optimizer to unequally spaced linear array synthesis with sidelobe level suppression and null control," IEEE Antennas and Wireless Propagation Letters, vol. 9, pp. 125-129, 2010.

[32] K. Guney and S. Basbug, "Seeker optimization algorithm for interference suppression of linear antenna arrays by controlling position-only, phase-only, and amplitude-only," International Journal of RF and Microwave Computer-Aided Engineering, vol. 21, no. 5, pp. 505-518, 2011.

[33] D. Mandal, S. P. Ghoshal, and A. K. Bhattacharjee, "Wide null control of symmetric linear antenna array using novel particle swarm optimization," International Journal of RF and Microwave Computer-Aided Engineering, vol. 21, no. 4, pp. 376382, 2011.

[34] S. Pal, A. Basak, and S. Das, "Linear antenna array synthesis with modified invasive weed optimisation algorithm," International Journal of Bio-Inspired Computation, vol. 3, no. 4, pp. 238-251, 2011.

[35] K. Guney and M. Onay, "Optimal synthesis of linear antenna arrays using a harmony search algorithm," Expert Systems with Applications, vol. 38, no. 12, pp. 15455-15462, 2011.

[36] M. A. Zaman and M. Abdul Matin, "Nonuniformly spaced linear antenna array design using firefly algorithm," International Journal of Microwave Science and Technology, vol. 2012, Article ID 256759, 8 pages, 2012.

[37] K. N. Abdul Rani, M. F. Abd Malek, and N. Siew-Chin, "Natureinspired cuckoo search algorithm for side lobe suppression in a symmetric linear antenna array," Radioengineering, vol. 21, no. 3, pp. 865-874, 2012.

[38] K. Kaur and V. K. Banga, "Synthesis of linear antenna array using firefly algorithm," International Journal of Scientific \& Engineering Research, vol. 4, pp. 601-606, 2013.

[39] B. Goswami and D. Mandal, "Nulls and side lobe levels control in a time modulated linear antenna array by optimizing excitations and element locations using RGA," Journal of Microwaves, Optoelectronics and Electromagnetic Applications, vol. 12, no. 2, pp. 238-255, 2013.

[40] K. Guney, A. Durmus, and S. Basbug, "Antenna array synthesis and failure correction using differential search algorithm," International Journal of Antennas and Propagation, vol. 2014, Article ID 276754, 8 pages, 2014.

[41] L. Pappula and D. Ghosh, "Linear antenna array synthesis using cat swarm optimization," AEU-International Journal of Electronics and Communications, vol. 68, no. 6, pp. 540-549, 2014.

[42] K. N. A. Rani, M. F. A. Malek, N. S. Chin, and A. A. Wahab, "Modified and hybrid cuckoo search algorithms via weightedsum multiobjective optimization for symmetric linear array geometry synthesis," International Journal of Advanced Research in Computer and Communication Engineering, vol. 3, pp. 67746781, 2014.

[43] K. Guney and S. Basbug, "Linear antenna array synthesis using mean variance mapping method," Electromagnetics, vol. 34, no. 2, pp. 67-84, 2014.

[44] P. Civicioglu, "Backtracking search optimization algorithm for numerical optimization problems," Applied Mathematics and Computation, vol. 219, no. 15, pp. 8121-8144, 2013. 
[45] P. Civicioglu, "Circular antenna array design by using evolutionary search algorithms," Progress in Electromagnetics Research B, no. 54, pp. 265-284, 2013.

[46] K. Guney, A. Durmus, and S. Basbug, "Backtracking search optimization algorithm for synthesis of concentric circular antenna arrays," International Journal of Antennas and Propagation, vol. 2014, Article ID 250841, 11 pages, 2014.

[47] A. Merzougui, A. Bonilla-Petriciolet, A. Hasseine, D. Laiadi, and N. Labed, "Modeling of liquid-liquid equilibrium of systems relevant for biodiesel production using Backtracking Search Optimization," Fluid Phase Equilibria, vol. 388, pp. 8492, 2015.

[48] A. El-Fergany, "Optimal allocation of multi-type distributed generators using backtracking search optimization algorithm," International Journal of Electrical Power \& Energy Systems, vol. 64, pp. 1197-1205, 2015.

[49] M. Modiri-Delshad and N. A. Rahim, "Solving non-convex economic dispatch problem via backtracking search algorithm," Energy, vol. 77, pp. 372-381, 2014.

[50] A. Askarzadeh and L. dos Santos Coelho, "A backtracking search algorithm combined with Burger's chaotic map for parameter estimation of PEMFC electrochemical model," International Journal of Hydrogen Energy, vol. 39, pp. 11165-11174, 2014.

[51] L. Wang, Y. Zhong, Y. Yin, W. Zhao, B. Wang, and Y. Xu, "A hybrid backtracking search optimization algorithm with differential evolution," Mathematical Problems in Engineering. In press. 

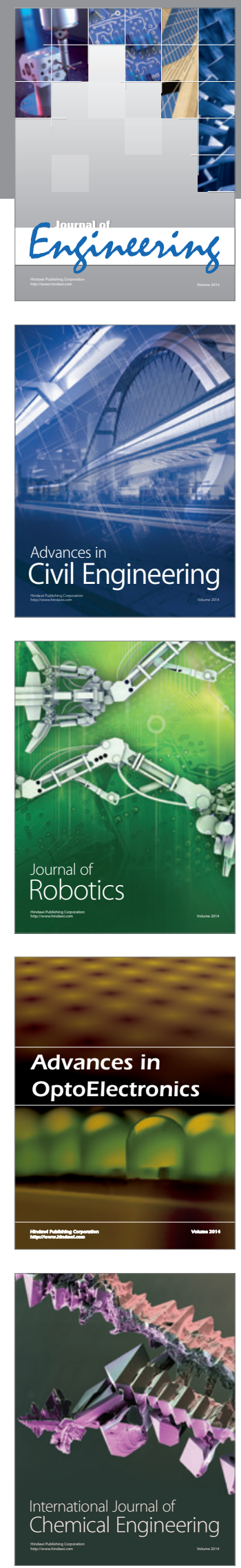

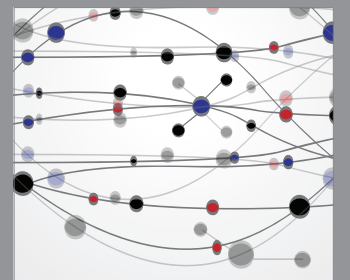

The Scientific World Journal
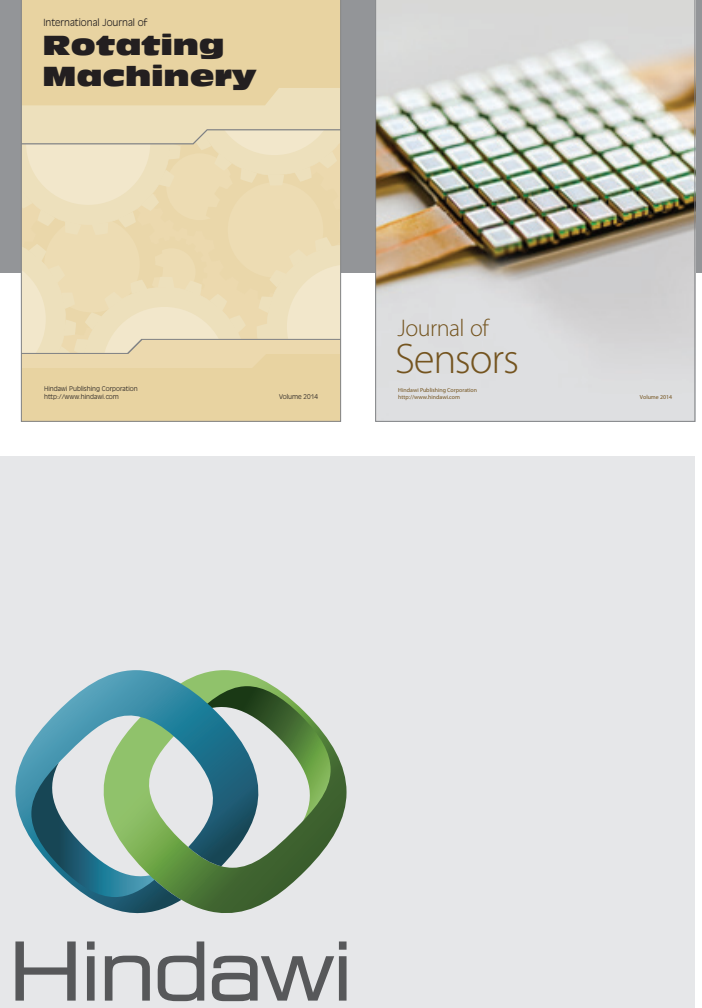

Submit your manuscripts at http://www.hindawi.com
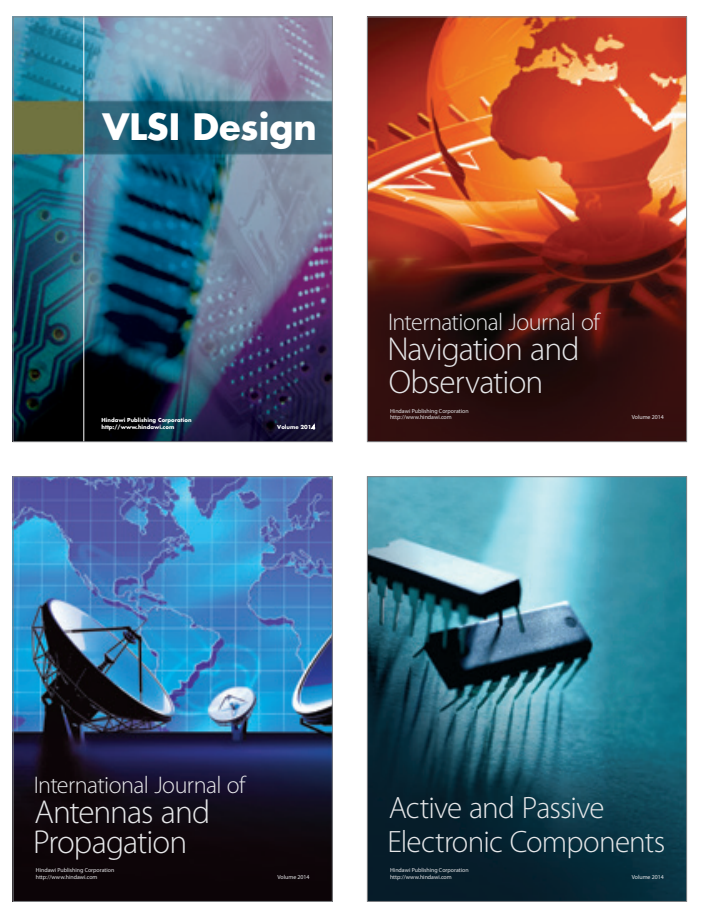
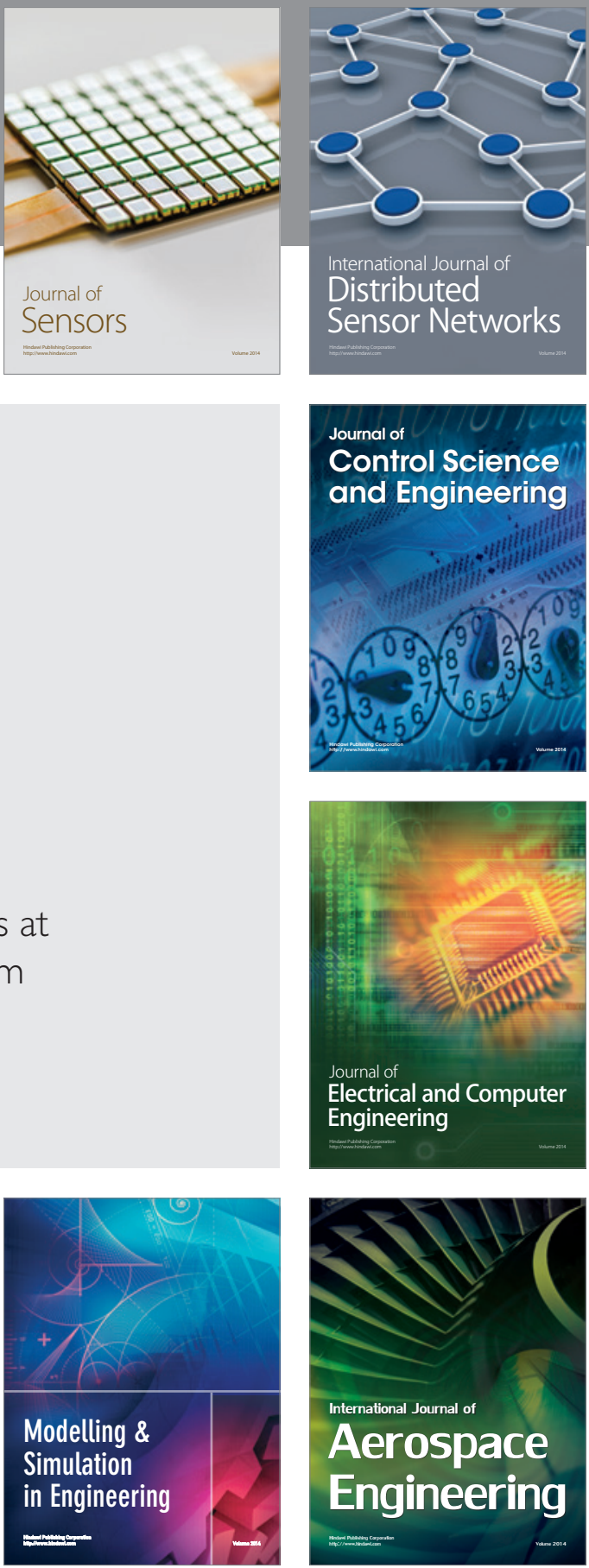

Journal of

Control Science

and Engineering
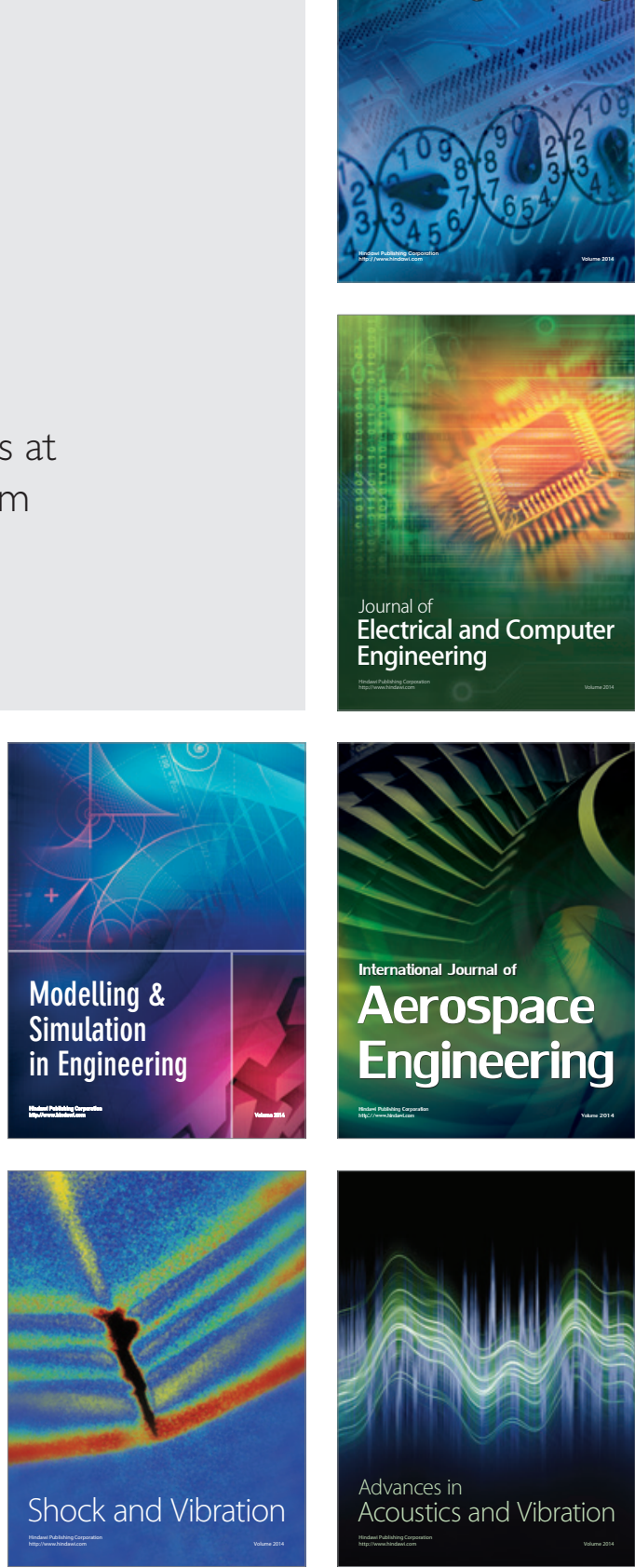